\title{
NIR spectroscopy coupled with multivariate data analysis in the prediction of the characteristics of mannitol lyophilized cakes
}

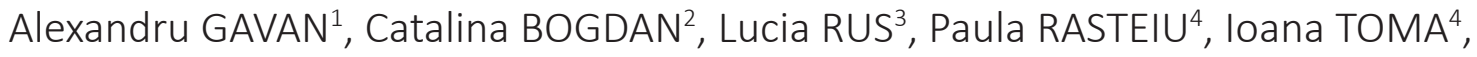 \\ Marcela ACHIM ${ }^{4}$, Sonia IURIAN ${ }^{4}$ \\ ${ }^{1}$ Department of Medical Devices, "Iuliu Hatieganu" University of Medicine and Pharmacy, \\ Cluj-Napoca, Romania
}

${ }^{2}$ Department of Dermopharmacy and Cosmetics, "Iuliu Hatieganu" University of Medicine and Pharmacy, Cluj-Napoca, Romania

${ }^{3}$ Department of Drug Analysis, "Iuliu Hatieganu" University of Medicine and Pharmacy, Cluj-Napoca, Romania

${ }^{4}$ Department of Pharmaceutical Technology and Biopharmacy, "Iuliu Hatieganu" University of Medicine and Pharmacy, Cluj-Napoca, Romania

\begin{abstract}
Mannitol is used in freeze-dried products as a bulking agent, consistency enhancer, or stabilizer, having the lowest hygroscopicity among the excipients commonly used as consistency agents, therefore it can be used in the formulation of unstable preparations. Lyophilization is a complex method of drying a solution at low temperature and low-pressure that addresses especially thermolabile substances, such as proteins. The end-product is a lyophilized powder, which can be administered parenterally after reconstitution with suitable solvents.

The objective of this study was to evaluate freeze-dried products obtained from mannitol solutions of different concentrations. Mannitol solutions of increasing concentrations of $2.5 \%, 5 \%, 7.5 \%$, and $10 \%$ were prepared by dissolving mannitol in distilled water. The prepared solutions were freeze-dried after a preliminary DSC analysis, in which the thermal phenomena that occurred during lyophilization were identified. Freeze-dried preparations were analyzed by different methods: macroscopic analysis by visual assessment and comparison with data from the literature, texture analysis by which several properties of these preparations were studied (hardness, deformation, mechanical work, adhesive strength, fracture resistance, and the number of fractures), evaluation of reconstitution time and porosity. The mannitol used in the lyophilization process, being partially amorphous, required differential calorimetric analysis to establish its glass transition and to avoid the collapse of the preparations during lyophilization. Finally, NIR spectroscopy was used to predict the characteristics of the freeze-dried powders in a non-invasive manner, without prior sample preparation.
\end{abstract}

Keywords: lyophilization, mannitol, NIR, MVDA

\section{INTRODUCTION}

Lyophilized products are the dosage forms of choice when the preparation of stable parenteral products is desired. Freeze-drying increases product stability, being one of the most used drying techniques $(1,2)$. It consists of three consecutive stages: freezing, primary, 
and secondary drying (3). Parenteral dosage forms regulations for excipients are strict, also the number of suitable excipients is limited (4). The freeze-dried products for parenteral administration meet the pharmacopeial requirements for the uniformity of dosage units, uniformity of content and uniformity of mass (5).

Bulking agents in lyophilized formulations are used for improving solubility and stability and promote controlled or prolonged drug delivery.

Mannitol represents one of the most frequently used bulking agents in lyophilization alongside glycine, lactose, and sucrose (2). It can exist in five different physical forms, out of which two of them (the amorphous mannitol and mannitol hemihydrate) are metastable and tend to transform into anhydrous crystalline forms during lyophilization or product shelf life (6). Mannitol needs to be in an amorphous form to have a stabilization effect as the long-term stability of the product can be affected by the incomplete crystallization during the freezing step (2).

Differential scanning calorimetry (DSC) is one of the most widely used analytical techniques applied to determine qualitative and quantitative information on the thermal properties of solid materials such as the melting and degradation temperatures, glass transition temperature, melt and crystallization enthalpy, specific and latent heats, and polymorphism $(7,8)$. The selection of sample mass, sample heating, and cooling rate control is critical for DSC test result accuracy (8). Another application of DSC is the evaluation of physicochemical interactions between the active pharmaceutical ingredient (API) and excipients to identify the most compatible combinations (9).

Near infrared (NIR) spectroscopy offers the chemical information from the spectral features and allows to inspect vials variability (1). The advantages offered by NIR spectroscopy are the possibility of continuously monitoring the chemical and physical characteristics during freeze-drying, in a non-invasive and nondestructive manner (10).

This study was performed following a request from the local pharmaceutical industry to formulate a freeze-dried generic product for parenteral delivery containing as active pharmaceutical ingredient a non-steroidal anti-inflammatory (NSAID). The original product contained mannitol as a filler, so this manuscript presents a preliminary study aiming to test the behaviour of freeze-dried mannitol solutions of different concentrations regarding their reconstitution capacity, mechanical properties, and porosity. Further, a NIR-chemometric method was developed to predict some of the physical characteristics using a noninvasive method, NIR spectroscopy coupled with multivariate data analysis (MVDA).

\section{MATERIALS AND METHODS}

\section{Materials}

Mannitol (Pearlitol $\left.{ }^{\circledR} 200 \mathrm{M}\right)$ was purchased from Merck \& Co (Darmstadt, Germany).

\section{Methods}

\section{Mannitol solution preparation}

$50 \mathrm{ml}$ of four mannitol solutions were prepared, of $2.5 \%, 5 \%, 7.5 \%$ and $10 \%(\mathrm{w} / \mathrm{V})$. They were divided into 25 vials of $2 \mathrm{ml}$ each.

\section{Differential scanning calorimetry (DSC)}

The DSC measurements were performed by a MettlerToledo (Mettler-Toledo GmbH, Greifensee, Switzerland) to determine the glass transition temperatures $\left(\mathrm{Tg}^{\prime}\right)$ and the crystallization events of the prepared solutions in their frozen states (before freeze-drying). From each of the liquid formulations, 15-25 mg were loaded into aluminum pans with pierced lids and cooled from 25 to $-55^{\circ} \mathrm{C}$ at a rate of $10^{\circ} \mathrm{C} / \mathrm{min}$, then reheated to $25^{\circ} \mathrm{C}$ at a rate of $20^{\circ} \mathrm{C} /$ $\min$.

\section{Freeze-drying}

For each mannitol solution, 15 samples of $2 \mathrm{ml}$ were taken using an automatic pipette and placed in lyophilized vials. Mannitol solutions were lyophilized using VirTis Advantage Plus (SP Scientific, Gardiner, NY, USA). A fast-freezing regime was applied to $-48^{\circ} \mathrm{C}$ at a speed of $1^{\circ} \mathrm{C} / \mathrm{min}$ (Figure 1 ). For the complete solidification of the product, this temperature of $-48^{\circ} \mathrm{C}$ was kept constant for 6 hours. The primary drying was performed at a temperature of $-25^{\circ} \mathrm{C}$, the pressure of 150 mTorr, for 40 hours. Secondary drying took place at a temperature of $20^{\circ} \mathrm{C}$, pressure of $350 \mathrm{mTorr}$, for 2.5 hours.

\section{Reconstitution time}

The reconstitution time was measured twice, first right after freeze-drying and second after one year of 


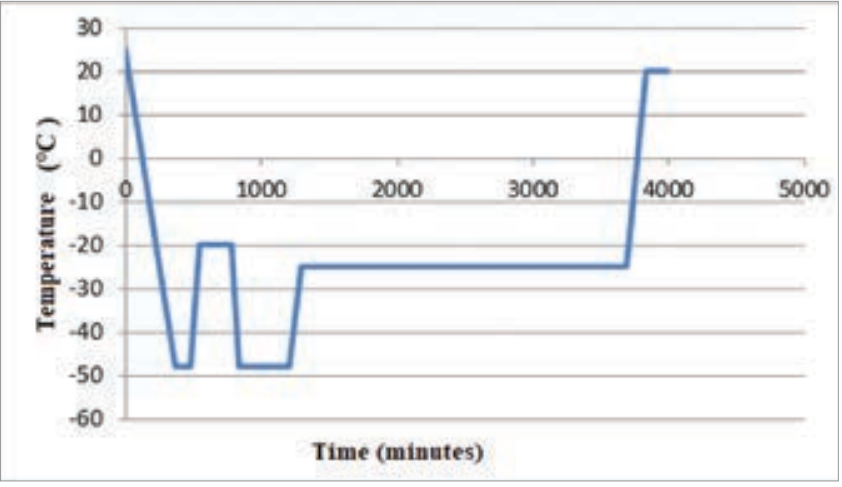

FIGURE 1. Freeze-drying process parameters

keeping in the desiccator at a temperature of $20 \pm 1^{\circ} \mathrm{C}$. The reconstitution time was determined manually by injecting the solvent into the lyophilized preparation vial. $2 \mathrm{ml}$ of distilled water was used for the reconstitution and the time necessary for the complete mannitol dissolution was recorded. A mean of five measurements and standard deviation (SD) were calculated.

\section{Porosity}

To calculate the porosity of the preparations, the diameter and height of the samples were measured. Using the average of the 2 measurements the sample volume was calculated with the following formula: $\left.V=\pi(d / 2)^{2} x h\right)$, where V-volume, $d$-diameter, h-height. The weight of the freeze-dried product was calculated by the difference of weight for each sample vial and the empty vials.

The density of the preparation was calculated with the formula: $\rho=m / V$, where $\rho$ - the density, $m$ - the weight of the freeze-dried product.

The porosity was determined from the equation $\varepsilon=(1-\rho) / \rho a$, where $\varepsilon$ - porosity and $\rho a=1.514 \mathrm{~g} / \mathrm{cm}^{3}$ - the true density of mannitol.

\section{Texture analysis}

Texture analysis was performed using Brookfield TexturePro CT V1.5 (Brookfield Engineering, SUA) equipped with an acrylic probe (TA10), directly into the freeze-drying vials. Constant pressure was applied at a load of $1 \mathrm{~g}$ and a speed of $0.2 \mathrm{~mm} / \mathrm{s}$, until a target distance of $7 \mathrm{~mm}$. Load vs. distance profiles were recorded for 5 samples of each concentration and several parameters were calculated: hardness, deformation, hardness work, adhesive strength, brittleness, and the number of fractures, as means of five measurements and standard deviations.

\section{NIR-spectroscopy}

A portable MicroNIR PAT-U (Viavi Solutions, California, SUA) spectrometer was used. The apparatus was equipped with Linear Variable Filter technology, which allows the reduction of the device's size and the direct measurement through the base of the vial. Spectra were recorded in reflectance mode, over the full range of the spectrometer, 950 to $1650 \mathrm{~nm}$, with a resolution of $6 \mathrm{~nm}$. Each spectrum represented the average of 200 scans, recorded with an integration time of $7 \mathrm{~ms} / \mathrm{scan}$. All parameters have been set using the device's own software, JDSU (California, USA). Five samples (vials) were evaluated from each concentration, five spectra being recorded directly through each lyophilized vial, resulting in a total of 100 spectra.

\section{MVDA}

The data was imported and analyzed using the SIMCA 14.0 software (Sartorius Stedim, Sweden). Principal Component Analysis (PCA) was performed to identify which spectral domain is specific for changes in the physical characteristics of the cake. The multivariate prediction model was developed using the Orthogonal Partial Least Squares (OPLS) method, which had the purpose to separate the $X$ - specific spectral systematic variation into predictive and orthogonal (uncorrelated) fractions (11).

\section{RESULTS AND DISCUSSION}

\section{Differential scanning calorimetry (DSC)}

Thermal events for $2.5 \%$ and $10 \%$ mannitol solutions were analysed by DCS. The thermogram of the $2.5 \%$ mannitol solution is presented in Figure 2 with an average $\mathrm{Tg}^{\prime}$ at $-29.36^{\circ} \mathrm{C}$, and the onset of $\mathrm{Tg}^{\prime}$ at $-30.96^{\circ}$ C. In Figure 3 the DSC thermogram of the $10 \%$ mannitol solution with an average $\mathrm{Tg}^{\prime}$ at $-29.36^{\circ} \mathrm{C}$ is presented, and the onset of $\mathrm{Tg}^{\prime}$ at $-30.55^{\circ} \mathrm{C}$.

Following the DSC analysis and the presented thermograms, it can be observed that at $-10^{\circ} \mathrm{Cthe}$ crystallization of mannitol solutions, which is an exothermic process, took place. After crystallization, the temperature drops to $-55^{\circ} \mathrm{C}$ where the preparation is completely frozen. Then the heating process has begun and at $-30^{\circ} \mathrm{C}$ the amorphous mannitol becomes glassy, a state in which the product had a lower viscosity. During the primary drying of lyophilization at this temperature the structure can collapse, due to the lack of rigidity. The primary drying should take 


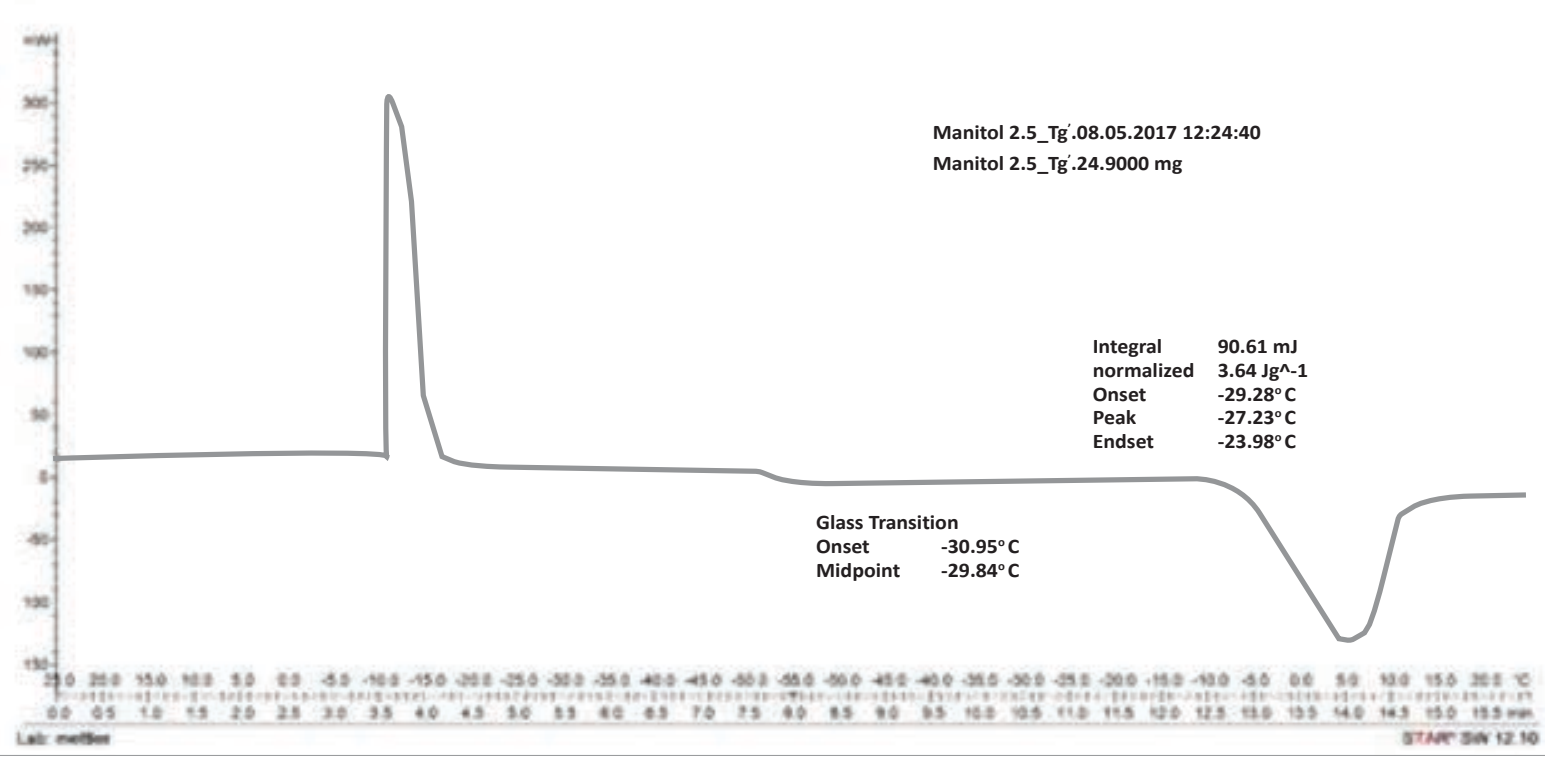

FIGURE 2. Thermogram of $2.5 \%$ mannitol solution

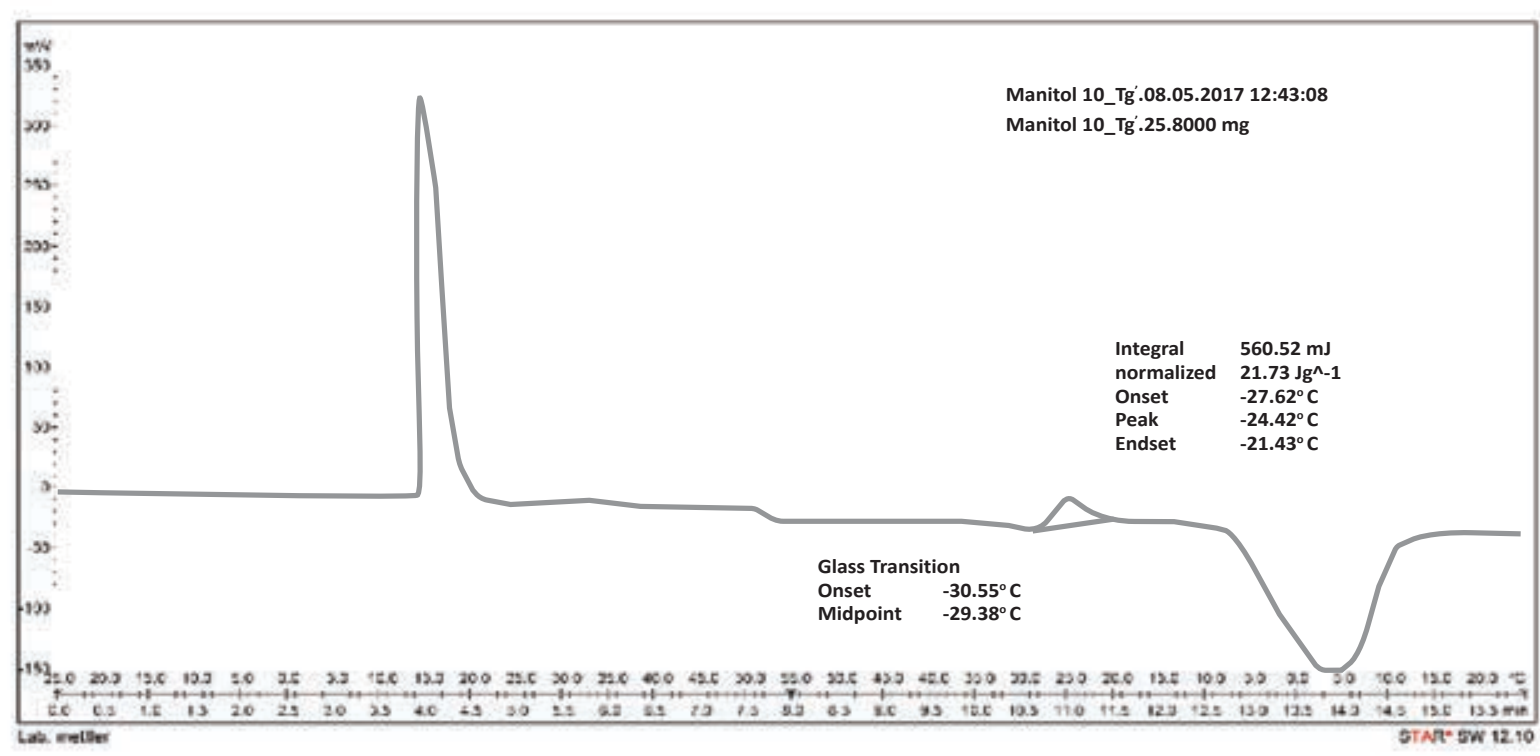

FIGURE 3. Thermogram of $10 \%$ mannitol solution

place at a lower temperature compared to the glass transition, when the structure is rigid, for example below $-32^{\circ} \mathrm{C}$. At such a low temperature, the primary drying proceeds much more slowly. As such, an annealing step was applied at $-20^{\circ} \mathrm{C}$ during the freezing stage, which would produce complete crystallization of mannitol and eliminate the glass transition (Figure 1). Therefore, a higher temperature, $-25^{\circ} \mathrm{C}$, could be chosen for the primary drying. Beyond the temperature of $-25^{\circ} \mathrm{C}$ the endothermic melting of the preparation begins.

\section{Reconstitution time}

Table 1 shows the reconstitution times obtained for each of the lyophilized products. The evaluation of the
TABLE 1. Reconstitution time of the freeze-dried products

\begin{tabular}{|c|c|c|}
\hline $\begin{array}{c}\text { Mannitol } \\
\text { concentration } \\
\text { (\%) }\end{array}$ & $\begin{array}{c}\text { Reconstitution time } \\
\mathbf{0}(\mathbf{s})\end{array}$ & $\begin{array}{c}\text { Reconstitution time } \\
\mathbf{1} \text { year (s) }\end{array}$ \\
\hline $2.5 \%$ & $16.574 \pm 1.063$ & $13.6 \pm 1.396$ \\
\hline $5 \%$ & $20.408 \pm 1.663$ & $17.236 \pm 1.642$ \\
\hline $7.5 \%$ & $33.89 \pm 3.993$ & $16.012 \pm 2.416$ \\
\hline $10 \%$ & $28.862 \pm 4.651$ & $21.78 \pm 2.264$ \\
\hline
\end{tabular}

reconstitution time was made immediately after preparation and after keeping the samples in the desiccator for 1 year. The results showed that increasing mannitol concentration, the time required for dissolution increases. In addition, significant 
TABLE 2. Porosity of the freeze-dried products

\begin{tabular}{|c|c|}
\hline $\begin{array}{c}\text { Mannitol concentration } \\
\text { (\%) }\end{array}$ & Porosity (g/cm $\left.{ }^{3}\right)$ \\
\hline $2.5 \%$ & $0.6442 \pm 0.0013$ \\
\hline $5 \%$ & $0.6296 \pm 0.0011$ \\
\hline $7.5 \%$ & $0.6132 \pm 0.00048$ \\
\hline $10 \%$ & $0.5971 \pm 0.0016$ \\
\hline
\end{tabular}

differences $(p<0.05)$ are observed between time 0 and time 1. Reconstitution is faster after holding in the desiccator, due to the elimination of the residual moisture.

\section{Porosity}

Table 2 shows the results of the porosity of the four freeze-dried products with different mannitol contents. The results showed that the $2.5 \%$ mannitol cake presented a more porous structure, whereas the $10 \%$ mannitol product showed the lowest porosity. As expected, increasing the solid content resulted in a porosity decrease. As high porosity or high surface area allows fast water immersion in the freeze-dried cake, it should have an impact on reconstitution behaviour. When comparing the two sets of results, the one for porosity with the reconstitution time, the correlation between the two parameters is revealed: high porosity facilitated reconstitution.

\section{Texture analysis}

The freeze-drying technique proved to be a major step in the development of stable parenteral products since the residual moisture is reduced, the stability of the formulation increases, and the reconstitution time is shortened due to the porous structure of the cake. At the same time, the brittle structure may be subjected to cracking and powdering during handling and transportation (12).

The application of the texture analysis technique to study the compressive mechanical properties of the lyophilized products directly in glass vials has been previously described as a sensitive and reliable method (13). Thus, the analysis of the intact cake may provide useful information about the mechanical strengths and texture properties such as tendency to fractures and elasticity. The analysis of the freezedried products directly in lyophilization vial avoids the distortion of the samples and water absorption during manipulation (13).

The load vs. time profiles of the four mannitol samples are presented in Figure 4. An increase of the load can be observed with the increase of the mannitol content in the lyophilized products.

The results showed an important influence of the mannitol content on the mechanical properties of the dried cakes. As shown in Table 3, the hardness increased while the mannitol concentration increased. There was an increase in the mechanical work required to apply the force as the mannitol concentration increased. The highest value of adhesiveness was obtained for the sample with 10\% mannitol and the lowest for the one with $5 \%$. The variation of the adhesiveness did not correlate with that of the mannitol content. The brittleness varied

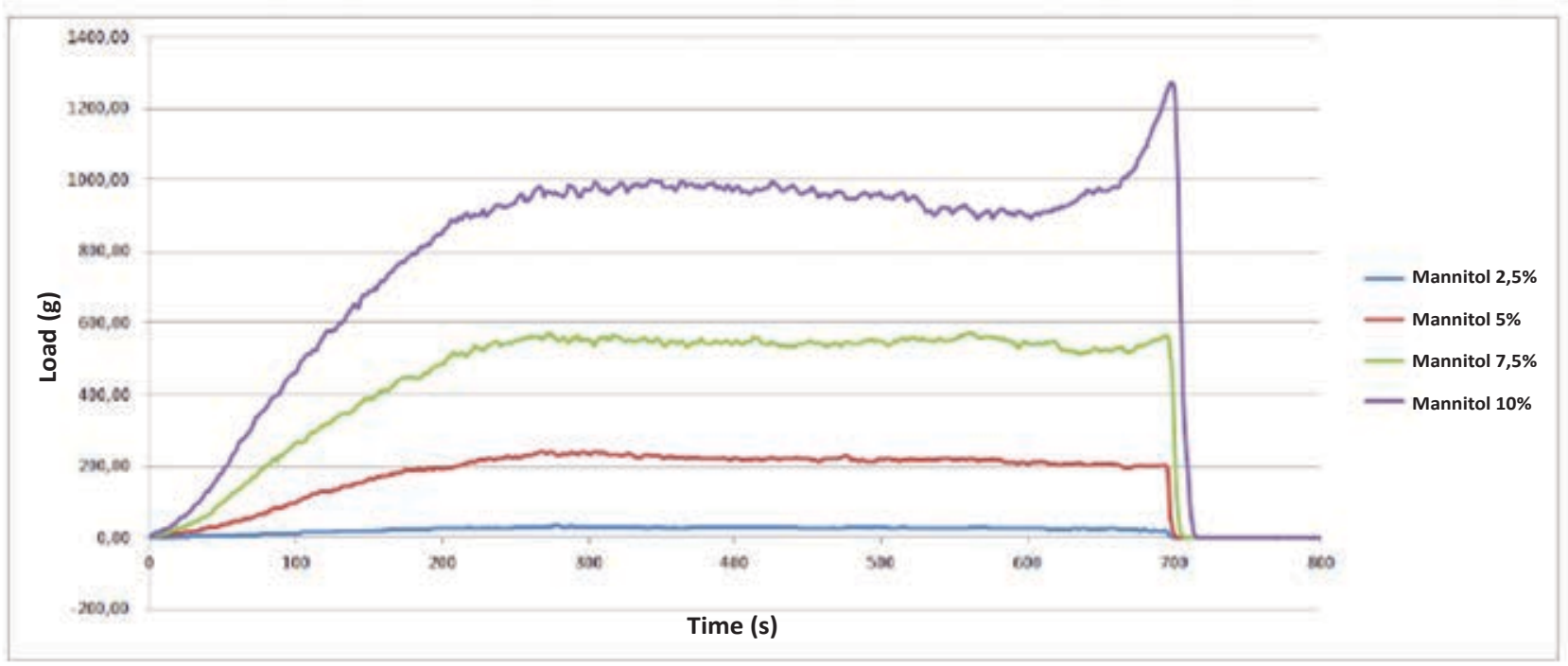

FIGURE 4. Texture profiles of lyophilized mannitol products 
TABLE 3. Parameters calculated from the texture analysis of lyophilized preparations

\begin{tabular}{|c|c|c|c|c|c|c|}
\hline Sample & Hardness $\mathbf{( g )}$ & $\begin{array}{c}\text { Deformation } \\
(\mathbf{m m})\end{array}$ & $\begin{array}{c}\text { Hardness work } \\
(\mathbf{m} \mathbf{)}\end{array}$ & $\begin{array}{c}\text { Adhesive force } \\
(\mathbf{g})\end{array}$ & $\begin{array}{c}\text { Brittleness (g) } \\
\text { fractures }\end{array}$ & $\begin{array}{c}\text { Number of } \\
\text { fracture }\end{array}$ \\
\hline Mannitol 2.5\% & $37.5 \pm 5.5$ & $2.8 \pm 0.24$ & $1.7 \pm 0.24$ & $2.9 \pm 1.1$ & $1.4 \pm 1.0$ & $149 \pm 7$ \\
\hline Mannitol 5\% & $253.7 \pm 58.2$ & $3.6 \pm 1.28$ & $12.5 \pm 3.08$ & $1.9 \pm 0.7$ & $40.6 \pm 40.1$ & $80 \pm 9$ \\
\hline Mannitol 7.5\% & $625.3 \pm 147.9$ & $4.9 \pm 2.20$ & $30.6 \pm 6.85$ & $2.6 \pm 0.8$ & $241.4 \pm 94.9$ & $66 \pm 7$ \\
\hline Mannitol 10\% & $1330.8 \pm 278.6$ & $6.7 \pm 0.65$ & $56.3 \pm 8.05$ & $4.0 \pm 0.8$ & $479.2 \pm 145.2$ & $47 \pm 17$ \\
\hline
\end{tabular}

proportionally to the hardness for samples with low concentrations of mannitol, high values of the number of fractures were obtained.

Mannitol showed its advantage to reinforce the structures of the lyophilized matrix, consistent with the results of other reports investigating the mechanical strength and the tendency to fractures of freeze-dried products $(14,15)$. Additionally, mannitol has been reported to improve the appearance of the cake by forming intact and porous structures, with superior appearance properties when compared to glucose, lactose, dextran 20, or sucrose (14).

\section{NIR-spectroscopy}

The spectral evaluation began with PCA, pursuing two objectives, to establish the most suitable spectral preprocessing method and to identify the spectral areas sensitive to changes in properties of interest i.e., mannitol concentration and cake porosity.
Several spectral preprocessing methods have been tested, over the whole registered spectral range as well as over the ranges representing the most spectral variation. By applying the 1st order Savitzky-Golay derivative baseline effects and linear trends were eliminated and the resolution of the spectra was improved. The model has been reduced to the spectral range representing the most significant variation, 1100 to $1300 \mathrm{~nm}$ (Figure 5). One more spectral domain, between 1450 and $1600 \mathrm{~nm}$, registered high variations in intensity. However, this domain did not reflect the properties of interest and did not improve the predictive performance, therefore it has not been included into the final model.

The statistical values reflecting the performance of the PCA model developed by considering the spectral range from 1100 to $1300 \mathrm{~nm}$ are presented in table 4 . The calculated cumulative R2X representing the data correlation was 0.969 , for a model with two main components explaining $50.5 \%$ and $46.4 \%$ of the spectral intensity variation.

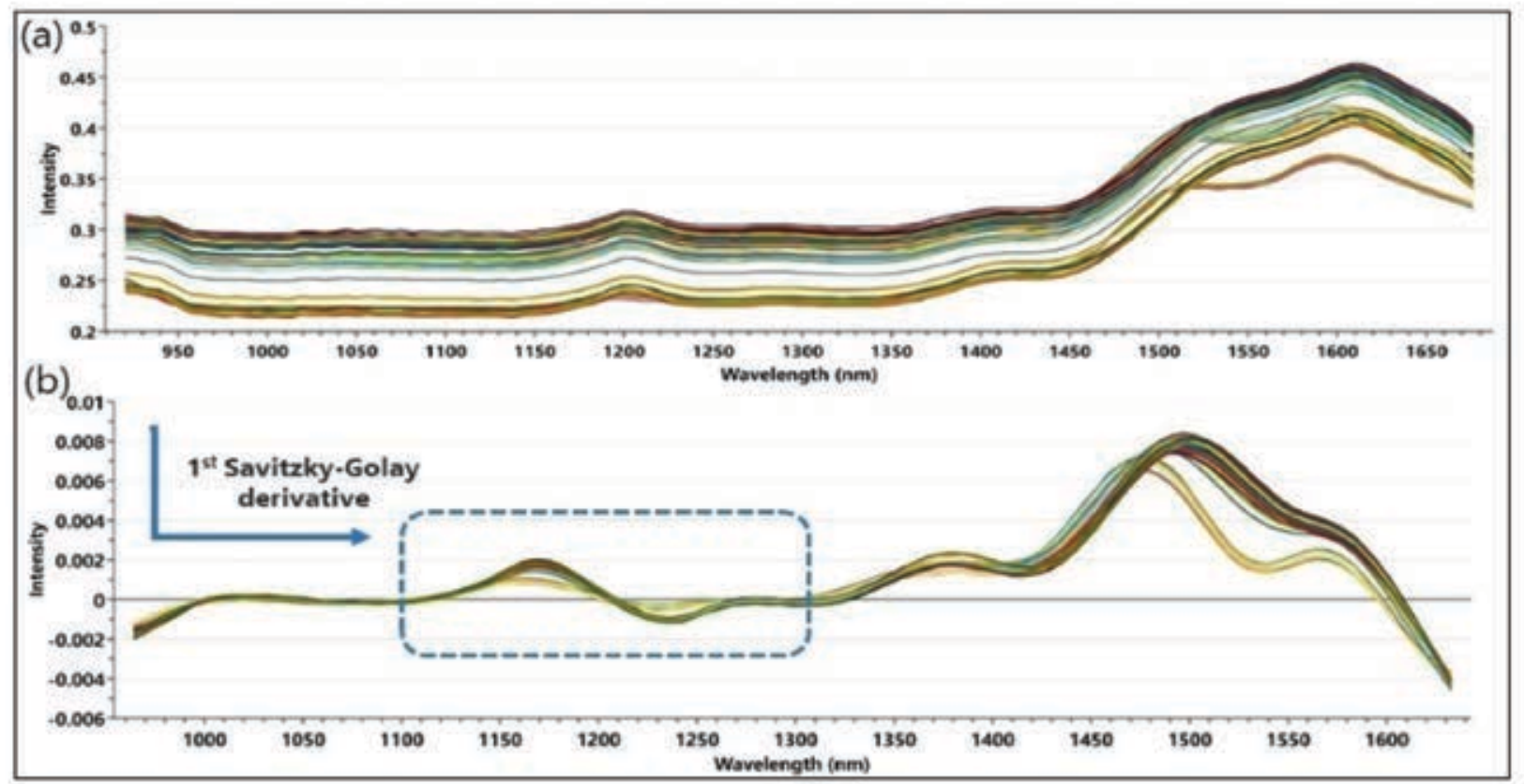

FIGURE 5. (a) - raw reflectance spectra, (b) - 1st Savitzky-Golay derivative pre-processed spectra 
ORIGINAL RESEARCH PAPERS

TABLE 4. PCA model parameters

\begin{tabular}{|c|c|c|c|c|c|}
\hline $\begin{array}{c}\text { Principal } \\
\text { components }\end{array}$ & $\mathbf{R}^{2} \mathbf{X}$ & $\mathbf{R}^{2} \mathbf{X}$ cumulative & Value & $\mathbf{Q}^{2}$ & $\mathbf{Q}^{2}$ cumulative \\
\hline 1 & 0.505 & 0.505 & 17.2 & 0.463 & 0.463 \\
\hline 2 & 0.464 & 0.969 & 15.8 & 0.936 & 0.966 \\
\hline
\end{tabular}

TABLE 5. Observed vs predicted mannitol concentration and porosity

\begin{tabular}{|c|c|c|c|c|c|}
\hline \multicolumn{3}{|c|}{ Concentration (\%) } & \multicolumn{3}{c|}{ Porosity (g/cm3) } \\
\hline Observed & Predicted & Recovery (\%) & Observed & Predicted & Recovery (\%) \\
\hline 2.5 & 2.562 & 102.5 & 0.644 & 0.644 & 100.0 \\
\hline 5.0 & 5.873 & 117.4 & 0.629 & 0.622 & 98.9 \\
\hline 7.5 & 7.567 & 100.9 & 0.613 & 0.612 & 99.8 \\
\hline 10.0 & 8.696 & 87.0 & 0.597 & 0.606 & 101.5 \\
\hline
\end{tabular}

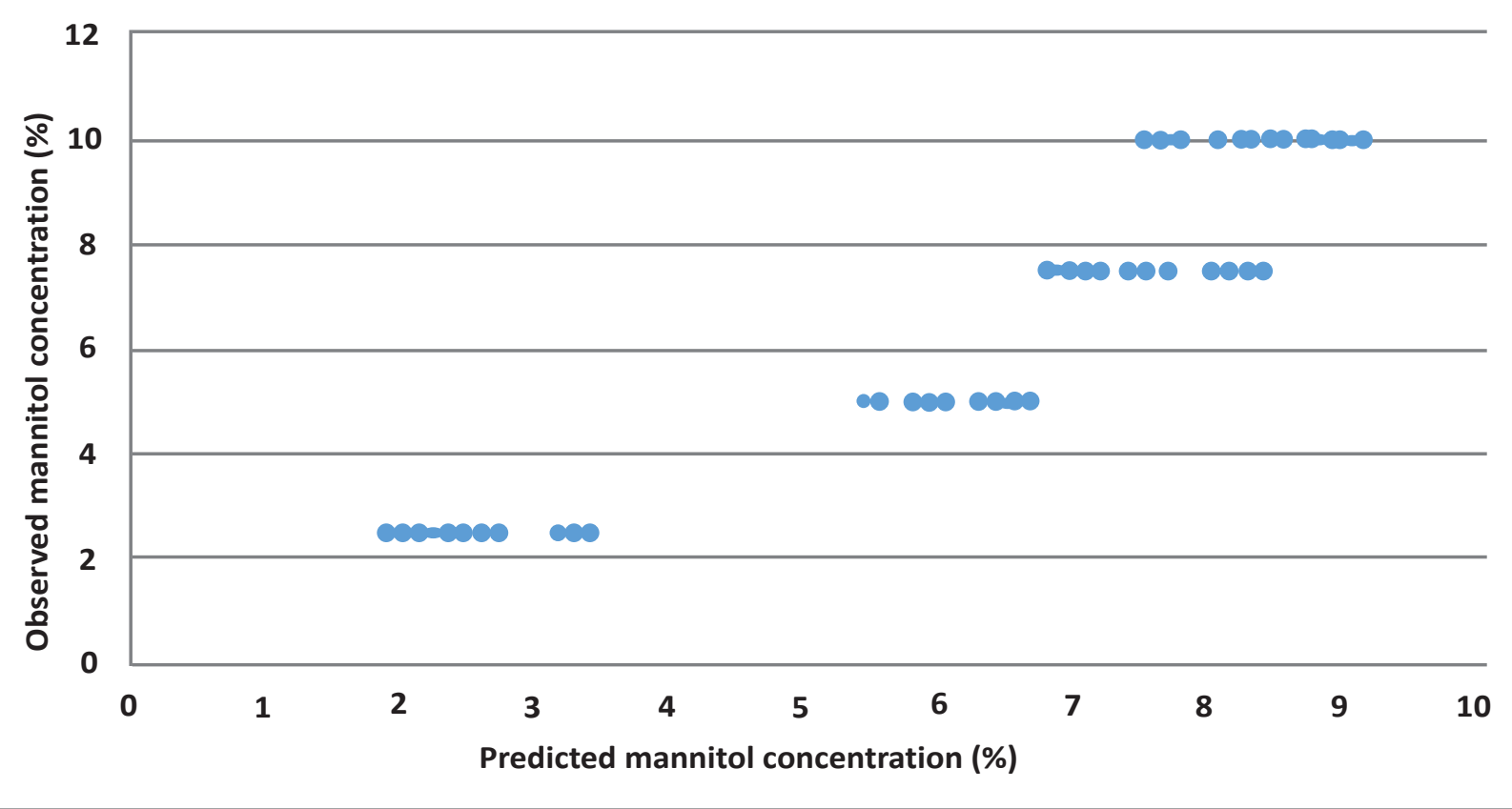

FIGURE 6. Observed vs. predicted - mannitol concentration

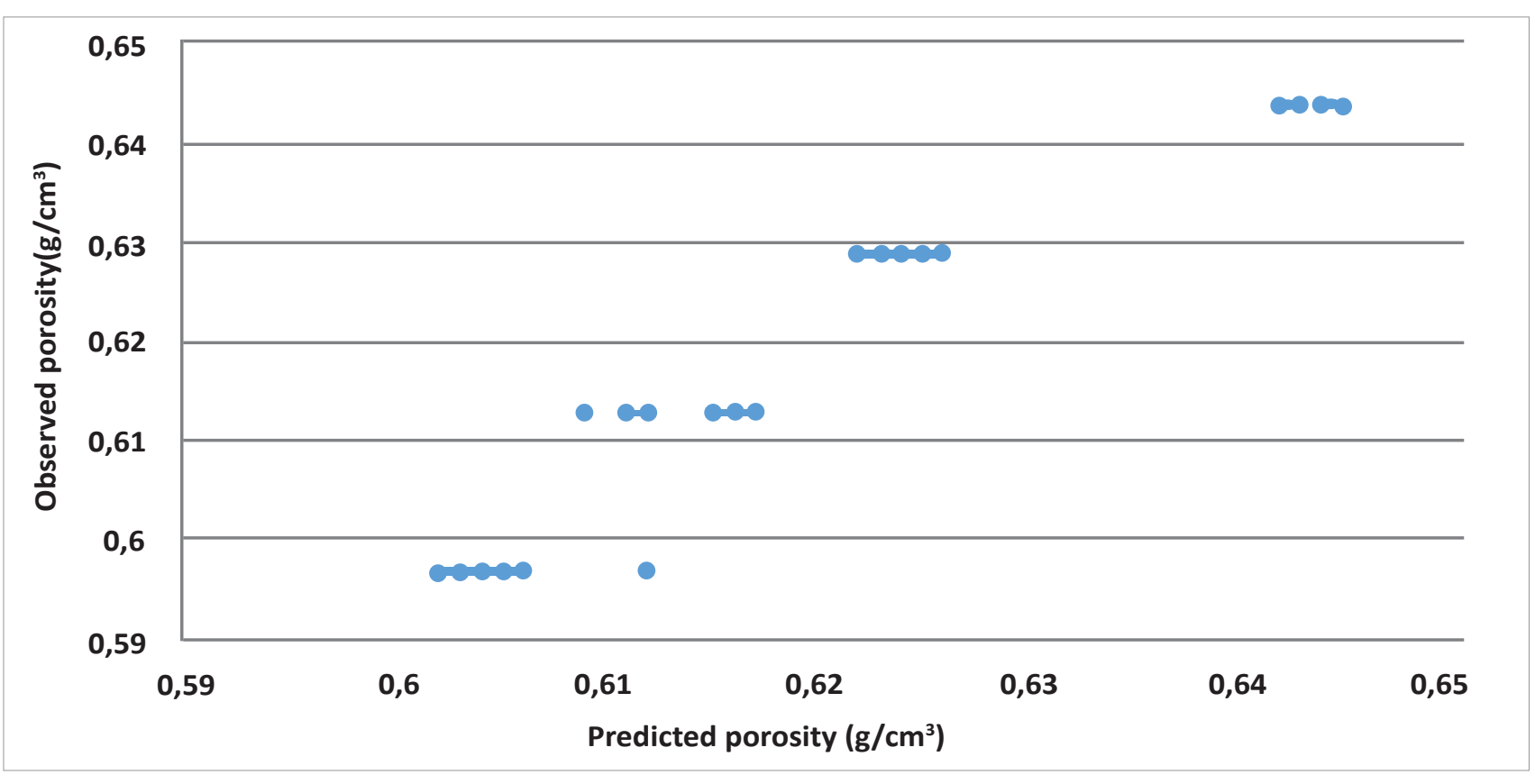

FIGURE 7. Observed vs. predicted - porosity 
The OPLS prediction model was developed with the purpose to estimate mannitol concentration and cake porosity, its defining parameters being presented in the Table 5. The predictive fraction of the model explaining $78 \%$ of the spectral variation, while the orthogonal (uncorrelated) fraction just $21.3 \%$, thus resulting a cumulative R2X of 0.993 .

The measured and NIR - predicted values of the two properties of interest are illustrated in the two following figures (Figure 6, Figure 7) and numerically presented in Table 6.

The cake's porosity prediction model delivered accurate results, the estimated values differing by a maximum of $1.5 \%$ units from the measured ones, for all sample concentrations.

Regarding the mannitol concentration prediction capacities of the NIR method, an overprediction can be observed for the $5 \%$ samples where the predicted values lay at an average of $5.8 \%$. Another model limitation can be observed for the most concentrated samples. The $10 \%$ mannitol solution is underpredicted at only $8.7 \%$. However, the prediction sets for all concentration were relatively precise, with relatively low coefficients of variation, of maximum $6.37 \%$, and no outliers.

The limitation of the technique's sensitivity can be explained by the fact that generally, the NIR absorption bands are wide, register weak intensity and overlap each other, also the spectra include physical and chemical information about all sample components. For this reason, multivariate data analysis and chemometric processing are almost always required to correlate the spectral domain with the samples' properties of interest. Even so, the data analysis does not always have the capacity to lead to the development of very sensitive NIR methods $(16,17)$.

One other fact inducing the methods limitations could be the spectral acquisition in reflectance mode. This measurement mode is typically applied for solid samples, as scattering and absorbance of the particles on the surface of the sample cause changes of the signal intensity. Besides the diffuse reflectance recording mode, as the low absorption coefficient of the NIR spectroscopy allows increased penetration of the sample, the direct analysis in transmittance would also be possible. This particular technique, performed by using other types of NIR spectrometers, allows the radiation beam to pass through the lyophilizate cake and could provide spectra containing more representative information (18). Therefore, a parallel reflectance and transmittance spectral analysis and prediction methods development could potentially make the subject of further comparative studies.

\section{CONCLUSIONS}

In this paper, the mechanical and physical properties of the lyophilized mannitol preparations were analyzed. Mannitol is the most used excipient in the preparation of lyophilized products as a diluent or stabilizer and was used as a model substance, ensuring an easy and quick restructuring of the finished product. The important influence of the mannitol concentration on the mechanical properties, the reconstitution time and the porosity were demonstrated following the performed analysis. The increase in mannitol concentration led to harder structures, with high fracture resistance, prolonged reconstitution time and low porosity. Finally, the NIR spectroscopy evaluation of lyophilized samples led to the development of a valid predictive model for estimating the concentration and porosity of lyophilized preparations.

\section{Acknowledgment}

This work was supported by a grant of the Romanian Ministry of Education and Research, CNCS-UEFISCDI, project number PN-III-P1-1.1-PD-2019-0795, within PNCDI III.

Authors Alexandru Gâvan, Cătălina Bogdan have equal contribution to the manuscript.

\section{Funding}

This work was supported by a grant of the Romanian Ministry of Education and Research, CNCS-UEFISCDI, project number PN-III-P1-1.1-PD-2019-0795, within PNCDI III. 


\section{REFERENCES}

1. Brouckaert D, De Meyer L, Vanbillemont B, Van Bockstal PJ, Lammens J, Mortier S, et al. Potential of Near-Infrared Chemical Imaging as Process Analytical Technology Tool for Continuous Freeze-Drying. Anal Chem. 2018;90(7):4354-62.

2. Butreddy A, Dudhipala N, Janga KY, Gaddam RP. Lyophilization of Small-Molecule Injectables: an Industry Perspective on Formulation Development, Process Optimization, Scale-Up Challenges, and Drug Product Quality Attributes. AAPS PharmSciTech. 2020;21(7):252.

3. Rayaprolu BM, Strawser JJ, Anyarambhatla G. Excipients in parenteral formulations: selection considerations and effective utilization with small molecules and biologics. Drug Dev Ind Pharm. 2018;44(10):1565-71.

4. Bjelošević $M$, Zvonar Pobirk $A$, Planinšek $O$, Ahlin Grabnar P. Excipients in freeze-dried biopharmaceuticals: Contributions toward formulation stability and lyophilisation cycle optimisation. Int J Pharm. 2020;576:119029.

5. European Pharmacopoeia. 10.5th ed. Strasbourg, Fance: Council of Europe; 2021.

6. Anko $M$, Bjelošević $M$, Planinšek $O$, Trstenjak $U$, Logar $M$, Ahlin Grabnar $\mathrm{P}$, et al. The formation and effect of mannitol hemihydrate on the stability of monoclonal antibody in the lyophilized state. Int J Pharm. 2019;564(January):106-16.

7. Leyva-Porras C, Cruz-Alcantar P, Espinosa-Sol V, Saavedra-Leos MZ. Application of Differential Scanning Calorimetry (DSC) and Modulated Differential Scanning. Polymers (Basel). 2019;12(5):1-21.

8. Sun X, Lee KO, Medina MA, Chu Y, Li C. Melting temperature and enthalpy variations of phase change materials (PCMs): a differential scanning calorimetry (DSC) analysis. Phase Transitions. 2018;91(6):667-80.

9. Rosasco MA, Bonafede SL, Faudone SN, Segall Al. Compatibility study of tobramycin and pharmaceutical excipients using differential scanning calorimetry, FTIR, DRX, and HPLC. J Therm Anal Calorim. 2018;134(3):1929-41.

10. Sylvester B, Porfire A, Van Bockstal PJ, Porav S, Achim M, Beer T $\mathrm{De}$, et al. Formulation Optimization of Freeze-Dried LongCirculating Liposomes and In-Line Monitoring of the FreezeDrying Process Using an NIR Spectroscopy Tool. J Pharm Sci. 2018;107(1):139-48.

11. Eriksson L, Byrne T, Johansson E, Trygg J, Vikstrom C. Multi- and megavariate data analysis. Basic principles and applications. 3rd ed. Sweeden: MKS Umetrics AB; 2013. 521.

12. Khairnar S, Kini R, Harwalkar M, Salunkhe K, Chaudhari SR. A Review on Freeze Drying Process of Pharmaceuticals. Int J Pharm Technol. 2013;4(1):76-94.

13. Hackl E V., Ermolina I. Using Texture Analysis Technique to Assess the Freeze-Dried Cakes in Vials. J Pharm Sci. 2016;105(7):2073-85.

14. Liu D, Galvanin F, Yu Y. Formulation Screening and Freeze-Drying Process Optimization of Ginkgolide B Lyophilized Powder for Injection. AAPS PharmSciTech. 2018;19(2):541-50.

15. Luo W-C, O’Reilly Beringhs A, Kim R, Zhang W, Patel SM, Bogner $\mathrm{RH}$, et al. Impact of Formulation on the Quality and Stability of Freeze-dried Nanoparticles. Eur J Pharm Biopharm. 2021 Oct 31 ;

16. De Beer T, Burggraeve A, Fonteyne M, Saerens L, Remon JP, Vervaet $C$. Near infrared and Raman spectroscopy for the in-process monitoring of pharmaceutical production processes. Int J Pharm. 2011;417(1-2):32-47.

17. Reich G. Near-infrared spectroscopy and imaging: Basic principles and pharmaceutical applications. Adv Drug Deliv Rev. 2005;57(8):1109-43.

18. Pasquini C. Near infrared spectroscopy: Fundamentals, practical aspects and analytical applications. J Braz Chem Soc. 2003;14(2):198-219. 